\title{
Lehren aus Vivian für den Gebirgswald - im Hinblick auf den nächsten Sturm
}

\author{
Raphael Schwitter \\ Arthur Sandri \\ Peter Bebi \\ Thomas Wohlgemuth \\ Peter Brang
}

\author{
Fachstelle für Gebirgswaldpflege $(\mathrm{CH})^{*}$ \\ Bundesamt für Umwelt $(\mathrm{CH})$ \\ Eidgenössische Forschungsanstalt für Wald, Schnee und Landschaft $(\mathrm{CH})$ \\ Eidgenössische Forschungsanstalt für Wald, Schnee und Landschaft $(\mathrm{CH})$ \\ Eidgenössische Forschungsanstalt für Wald, Schnee und Landschaft $(\mathrm{CH})$
}

\begin{abstract}
Lehren aus Vivian für den Gebirgswald - im Hinblick auf den nächsten Sturm
Der Wintersturm «Vivian» vom Februar 1990 überraschte die schweizerische Forstwirtschaft durch sein enormes Ausmass und konfrontierte sowohl Forscher als auch Praktiker mit ganz neuen Fragen und Herausforderungen. 24 Jahre nach dem Sturm beurteilten die Mitglieder der Schweizerischen Gebirgswaldpflegegruppe anlässlich zweier Arbeitstagungen den Verlauf der Wiederbewaldung und die Entwicklung der Schutzwirkung in mehreren gut dokumentierten, hoch gelegenen Viviansturmflächen in den Kantonen St. Gallen und Graubünden. Der Entscheid, ob das Holz auf solchen Sturmflächen belassen oder geräumt werden soll, hängt vor allem von der Beurteilung des Risikos für Borkenkäferbefall ab. Liegendes Holz kann vorübergehend die Risiken durch Naturgefahrenprozesse reduzieren und auch die Wiederbewaldung begünstigen, indem es Schneebewegungen vermindert und längerfristig Moderholz bildet. Auf einigen der beurteilten Flächen ist die Wiederbewaldung bereits so weit fortgeschritten, dass die Schutzwirkung nahezu wiederhergestellt ist. Auf der Mehrzahl der Flächen ist dies jedoch auch nach 24 Jahren noch nicht der Fall. Ist im Altbestand schon Verjüngung vorhanden, beschleunigt diese als sogenannte Vorverjüngung die Wiederbewaldung der Sturmfläche erheblich. Wo die Vorverjüngung fehlt, kann durch Pflanzung ein wesentlicher zeitlicher Vorsprung gegenüber der sich erst allmählich einstellenden Naturverjüngung erreicht werden. Zusammenfassend kann festgehalten werden, dass in Hochlagen auf grossen Sturmflächen ohne Vorverjüngung das Potenzial für die Naturverjüngung begrenzt ist und dass bei zukünftigen Windwürfen die verschiedenen Handlungsoptionen noch differenzierter kombiniert werden sollten als bisher.
\end{abstract}

Keywords: windthrow gaps, natural regeneration, planting, salvage logging, protection forest doi: $10.3188 /$ szf.2015.0159

* c/o Bildungszentrum Wald, Bovel, Postfach 52, CH-7304 Maienfeld, E-Mail raphael.schwitter@ibw.ch
S türme wie «Vivian» (1990) oder «Lothar» (1999) werden immer wieder Waldschäden verursachen und die verantwortlichen Bewirtschafter vor schwierige Entscheidungen stellen. Daher beschäftigten sich rund 40 Mitglieder der Schweizerischen Gebirgswaldpflegegruppe (GWG) 24 Jahre nach «Vivian» anlässlich zweier Arbeitstagungen mit den Lehren aus einer langjährigen Forschungs- und Beobachtungstätigkeit auf solchen Sturmflächen (GWG 2014). In diesem Beitrag stellen wir die Entscheidungssituation im Jahr 1990 nach dem Sturm Vivian dar und schälen aufgrund der Tagungsergebnisse rückblickend heraus, welche Entscheidungskriterien sich für die Behandlung von Sturmflächen als besonders wichtig erwiesen. Wir beurteilen für 16 Totalschadenflächen mit unterschiedlicher Behandlung, wie weit die Schutzwirkung 24 Jahre nach Vivian genügt und ob diesbezüglich weiterer Hand- lungsbedarf besteht. Zum Abschluss stellen wir die noch offenen Fragen dar.

\section{Vivian im Rückblick: eine grosse Herausforderung für die Forstleute}

Der Wintersturm Vivian vom 26. bis 28. Februar 1990 brachte der schweizerischen Forstwirtschaft in mehrfacher Hinsicht ein böses Erwachen. Der sogenannte grossflächige Zusammenbruch, der während Jahrzehnten als schlimmstmögliches Resultat vernachlässigter Waldpflege gehandelt worden war, war über Nacht Tatsache geworden - allerdings wurden durch die Wucht des Sturmes auch gepflegte Waldbestände zerstört. Die Forstleute waren mit Sturmwürfen von bisher unbekanntem Ausmass konfrontiert und mussten sich eingestehen, 
tellandes lag. Die Hauptfragen blieben allerdings die gleichen: räumen oder belassen? Pflanzen oder allein auf Naturverjüngung setzen? Borkenkäfer bekämpfen oder nicht? Antworten auf diese Fragen gab die in einer provisorischen Fassung vorliegende «Entscheidungshilfe bei Sturmschäden im Wald» (BAFU 2008), ein Produkt der Sturmschadenforschung, das da und dort zum Einsatz kam. Gleichzeitig konnten einige Kantone und viele Forstleute auf ihre Erfahrungen mit der Bewältigung von Vivian zurückgreifen. Insgesamt war der Umgang mit den Sturmschäden nach 1999 gelassener als nach 1990, und die Diskussionen über Räumen oder Belassen wurden weniger heftig geführt. Die Sturmfolgen konnten auch mit einem geringeren Aufwand an Bundesmitteln bewältigt werden, obschon die Sturmholzmenge viel höher war. Trotzdem beruhten noch viele Entscheide auf unsicheren Grundlagen. Die Ereignisanalyse Lothar (WSL \& BUWAL 2001) wies denn auch noch auf einen beträchtlichen Forschungsbedarf hin.

\section{Versuchsflächen für Wissenschaft und Praxis}

Im Jahr 1990 war das Anlegen von grossflächigen Versuchen mit unterschiedlichen und teils unkonventionellen Behandlungsvarianten eine Herausforderung, mussten doch neben Behörden und Bevölkerung zum Teil auch noch Vorgesetzte und Berufskollegen überzeugt werden. Vor allem das Argument, dass mit dem liegen gelassenen Holz eine Borkenkäferzucht betrieben werde, war schwierig zu entkräften; dazu kam die Unsicherheit, ob denn die Wiederbewaldung tatsächlich rasch genug ablaufen würde (Schwitter 1996).

Theoretisch standen mit den Alternativen «Räumen» oder «Belassen», «Pflanzen» oder «Auf Naturverjüngung setzen» und «Verbauen» oder «Nicht verbauen» acht verschiedene Behandlungsoptionen zur Verfügung. Doch drei davon wurden vorgängig ausgeschlossen. So wird die Kombination von Räumen, Verbauen und Naturverjüngung in der Praxis

\begin{tabular}{|c|c|c|c|c|c|c|}
\hline Nr. & $\begin{array}{l}\text { Kanton, } \\
\text { Objektbezeichnung }\end{array}$ & Koordinaten & $\begin{array}{l}\text { Höhe ü. M. } \\
\text { (m) }\end{array}$ & Exposition & Standorttyp & $\begin{array}{l}\text { Behandlung und weitere } \\
\text { Merkmale }\end{array}$ \\
\hline $3^{* *}$ & SG, Pfäfers, Grot & $757000 / 204300$ & 1520 & W & $\begin{array}{l}\text { Buntreitgras-(Tannen)-Fichtenwald } \\
\left(60^{*}\right)\end{array}$ & $\begin{array}{l}\text { Streuschäden, verbaut (Drei- } \\
\text { beinböcke), bepflanzt }\end{array}$ \\
\hline $6^{*}$ & $\begin{array}{l}\text { SG, Pfäfers, } \\
\text { Haselhühneregg }\end{array}$ & $756700 / 202750$ & 1470 & W & Farnreicher Tannen-Buchenwald (20) & Geräumt \\
\hline $7^{*}$ & $\begin{array}{l}\text { SG, Pfäfers, } \\
\text { Matongatter }\end{array}$ & $756600 / 202400$ & 1500 & NW & Hochstauden-Tannen-Fichtenwald (50) & Belassen \\
\hline $10^{* *}$ & $\begin{array}{l}\text { GR, Curaglia, Tschaler } \\
\text { Ost }\end{array}$ & $709350 / 169550$ & 1670 & NO & $\begin{array}{l}\text { Alpenlattich-Fichtenwald mit Wollreit- } \\
\text { gras (57C) }\end{array}$ & Geräumt \\
\hline $11^{*}$ & $\begin{array}{l}\text { GR, Disentis, Cavorgia } \\
\text { Nord }\end{array}$ & $707320 / 171040$ & 1450 & NW & Alpendost-Tannen-Fichtenwald (50) & Geräumt \\
\hline $12^{*}$ & $\begin{array}{l}\text { GR, Disentis, Cavorgia } \\
\text { Mitte }\end{array}$ & $707240 / 170940$ & 1450 & NW & Alpendost-Tannen-Fichtenwald (50) & Belassen \\
\hline $13^{*}$ & $\begin{array}{l}\text { GR, Disentis, Cavorgia } \\
\text { Süd }\end{array}$ & $707180 / 170850$ & 1450 & NW & Alpendost-Tannen-Fichtenwald (50) & Geräumt, bepflanzt \\
\hline $14^{* *}$ & $\begin{array}{l}\text { GR, Tujetsch, Uaul } \\
\text { Bugnei oben }\end{array}$ & $703480 / 171400$ & 1730 & $S$ & Typischer Preiselbeer-Fichtenwald (58) & Geräumt, verbaut, bepflanzt \\
\hline
\end{tabular}

Tab 1 An der Tagung der Schweizerischen Gebirgswaldpflegegruppe beurteilte Sturmflächen. Bei den massgebenden Gefahrenprozessen handelt es sich bei allen Objekten um Lawinen, teilweise überlagert durch Gerinne- und Rutschungsprozesse. Der Standorttyp richtet sich nach Frehner et al (2005). *Versuchsflächen der WSL, ** Beobachtungsflächen der Praxis. 


\begin{tabular}{|c|c|c|c|}
\hline Massnahmen & Naturverjüngung & Pflanzung & Verbau \\
\hline \multirow[t]{2}{*}{ Belassen } & $\begin{array}{l}\text { Pfäfers, Matongatter (7) } \\
\text { Disentis, Cavorgia Mitte (12) }\end{array}$ & Pfäfers, Tischli (4) & - \\
\hline & \multicolumn{3}{|c|}{ Pfäfers, Grot (Streuschäden mit Stützpunktpflanzungen und Dreibeinböcken; 3) } \\
\hline \multirow[t]{2}{*}{ Räumung } & \multirow{2}{*}{$\begin{array}{l}\text { Pfäfers, Höfwald (2) } \\
\text { Pfäfers, Haselhühneregg (6) } \\
\text { - Curaglia, Tschaler Ost (10) } \\
\text { Disentis, Cavorgia Nord (11) } \\
\text { Tujetsch, Val Bugnei (16) }\end{array}$} & $\begin{array}{l}\text { Pfäfers, Chimichopfwald (1) } \\
\text { - Curaglia, Tschaler West (9) } \\
\text { Disentis, Cavorgia Süd (13) }\end{array}$ & - \\
\hline & & $\begin{array}{l}\text { Pfäfers, Bläserberg (5) } \\
\text { Curaglia, Stavel (8) } \\
\text { Tujetsch, Uaul Bugnei oben (14) } \\
\text { Tujetsch, Uaul Bugnei unten (15) }\end{array}$ & \\
\hline
\end{tabular}

Tab 2 Gutachtliche Beurteilung der Entwicklung der Wiederbewaldung und der Schutzwirkung 24 Jahre nach dem Sturm Vivian. Grün: Schutzwirkung ganz oder nahezu wiederhergestellt, rot: Schutzwirkung noch deutlich ungenügend, schwarz: Flächen mit spezieller Situation. Die Zahlen in Klammern entsprechen der Objektnummerierung in Tabelle 1.

kaum angewendet, da die Pflanzkosten im Vergleich zu den Kosten für den Verbau sehr gering und zusätzliche Pflanzungen als Ergänzung zur Naturverjüngung sehr wirksam sind. Ebenso ist die Kombination aus Belassen und Verbauen - ob mit oder ohne Pflanzung - unpraktikabel, weil das Errichten von Verbauungen die Räumung der Fläche voraussetzt.

Jede der verbleibenden fünf Kombinationen ist in den 16 Sturmflächen, die an der Tagung der GWG im August 2014 beurteilt wurden, mit mindestens einem Beispiel vertreten (Tabelle 1). Auf den sechs WSL-Flächen wurden von 1992 bis 2010 acht Datenerhebungen durchgeführt (Brang et al 2015, dieses Heft). Diesen gut dokumentierten Flächen stehen zehn Beobachtungsflächen gegenüber, welche durch eine Beschreibung der Anfangssituation charakterisiert sind. Die Versuchs- und Beobachtungsflächen dienen heute als Beispiele für die gewählten Behandlungsvarianten. Die Auswirkungen der vor 25 Jahren getroffenen Entscheidungen können auf den Flächen veranschaulicht und im Licht der eigenen Erfahrungen reflektiert werden.

\section{Wichtige Entscheidungskriterien zur Behandlung von Sturmschadenflächen}

An ihrer Tagung vom August 2014 beurteilten die Mitglieder der GWG die 16 Objekte in Gruppenarbeiten. Sie stützten sich dabei auf die Entscheidungshilfe bei Sturmschäden im Wald (BAFU 2008) und stellten die massgebenden Entscheidungskriterien fest. Die Objekte umfassen Standorte der obermontanen, hochmontanen und subalpinen Stufe, wobei als Naturgefahr vor allem Lawinenprozesse zu erwarten sind.

Die Diskussionen zeigten, dass der Grundsatzentscheid «Räumen» oder «Belassen» in diesen hoch gelegenen Flächen im Wesentlichen vom Umgang mit der Borkenkäferproblematik abhängt. Die Option «Belassen» ist nur bedingt mit der konsequenten Bekämpfung der Borkenkäfer vereinbar. Wegen fehlender Grundlagen wurde das Sturmholz nach Vivian nur ausnahmsweise liegen gelassen. Auch nach Lothar löste der Entscheid, auf die Käferbekämpfung zu verzichten beziehungsweise Sturmholz liegen zu lassen, Kontroversen aus.

Das Kriterium Kostenanalyse wird an den jeweils aktuellen Erntekosten im Vergleich zum Holzerlös gemessen. Ist eine kostendeckende Nutzung möglich, steigt der Anreiz für die Waldeigentümer, das Holz zu räumen, ungeachtet der längerfristigen Auswirkungen einer Räumung auf die Entwicklung der Sturmfläche. Die öffentlichen Beiträge zur Sturmschadenbehebung verstärkten diesen Anreiz zusätzlich.

In der Praxis standen somit die Kriterien Borkenkäfer und Erntekosten oft einer objektiven Beurteilung der verschiedenen Optionen im Hinblick auf die Naturgefahrenprozesse und die Wiederbewaldung im Wege. Einige der an der Tagung beurteilten Flächen zeigen jedoch, dass liegen gelassenes Holz den Verlauf der Wiederbewaldung günstig beeinflussen kann, indem es gegen Gleitschnee schützt und langfristig ein geeignetes Verjüngungssubstrat bildet; liegen gelassenes Holz kann auch kostspielige Verbauungen ersetzen. In der Zeit seit 1990 sind in den belassenen Flächen im Gegensatz zu sauber geräumten Flächen keine Lawinen angerissen, und auch sonst wurden in belassenen Flächen nur wenige Naturgefahrenprozesse beobachtet (Bebi et al 2015, dieses Heft).

\section{Schutzwirkung nach 24 Jahren erst teilweise wiederhergestellt}

Die gutachtliche Beurteilung der 16 Objekte bezog sich auf die ausgeführten Massnahmen beziehungsweise die Unterlassungen und deren Auswirkungen auf die Entwicklung der Wiederbewaldung und der Schutzwirkung 24 Jahre nach dem Sturm. Damit die Schutzwirkung als ganz oder nahezu wiederhergestellt betrachtet wurde, musste auf der Flä- 
che ein Jungwald in der beginnenden Stangenholzstufe mit einem Deckungsgrad von 50\% und einem ausreichenden Anteil an immergrünen Nadelbäumen vorhanden sein (Frehner et al 2005). Für fünf Objekte fiel diese Beurteilung positiv aus, für sieben war das Resultat unbefriedigend, und in vier Objekten liegt eine spezielle Situation vor (Tabelle 2).

\section{Die Entwicklung begünstigende Faktoren}

$\mathrm{Zu}$ den fünf Objekten mit erfolgreicher Entwicklung gehören in erster Linie einige derjenigen, die geräumt, verbaut und bepflanzt worden waren. Das entspricht dem traditionellen Vorgehen für wichtige Schutzwälder. Ein solches Resultat ist nach 24 Jahren allerdings nur auf relativ wüchsigen Standorten, bei ausreichender Bepflanzung sofort nach der Räumung und bei tragbarem Wildeinfluss erreichbar, zum Beispiel beim Objekt «Stavel» in Curaglia (Abbildung 2, Tabelle 2). Im Gegensatz dazu müssen auf der Fläche «Bläserberg» die Holzschneerechen zum Teil erneuert werden, weil sich auf diesem steilen und flachgründigen Standort die Verjüngung nur langsam entwickelt.

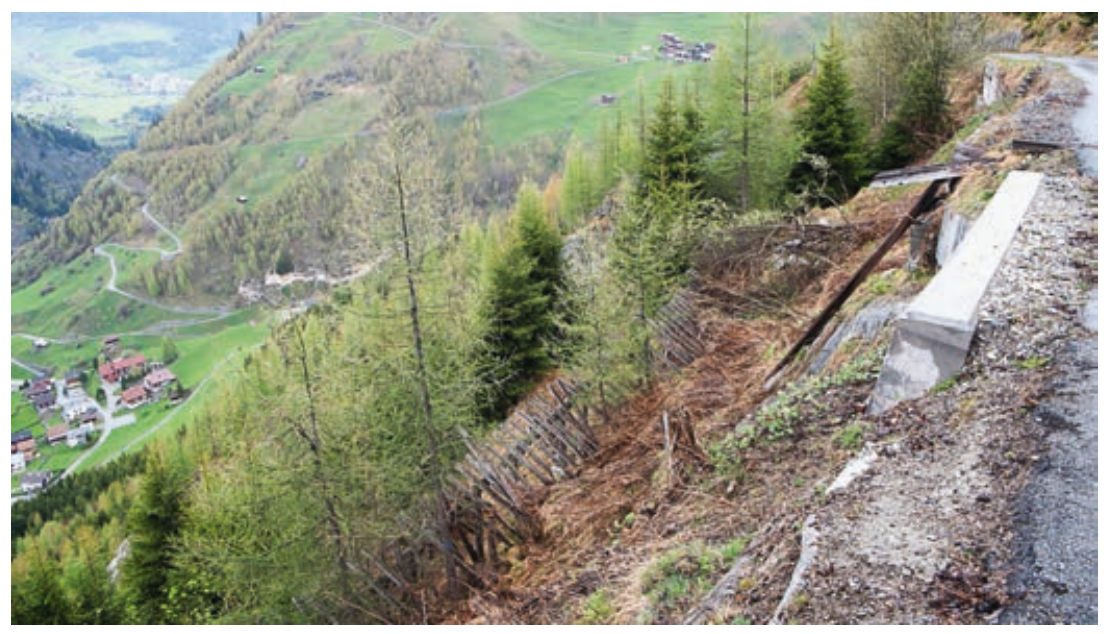

Abb 2 Die geräumte, verbaute und bepflanzte Fläche Curaglia, Stavel, am 7. Mai 2014: 24 Jahre nach dem Sturm ist die Schutzwirkung nahezu wiederhergestellt. Foto: Raphael Schwitter

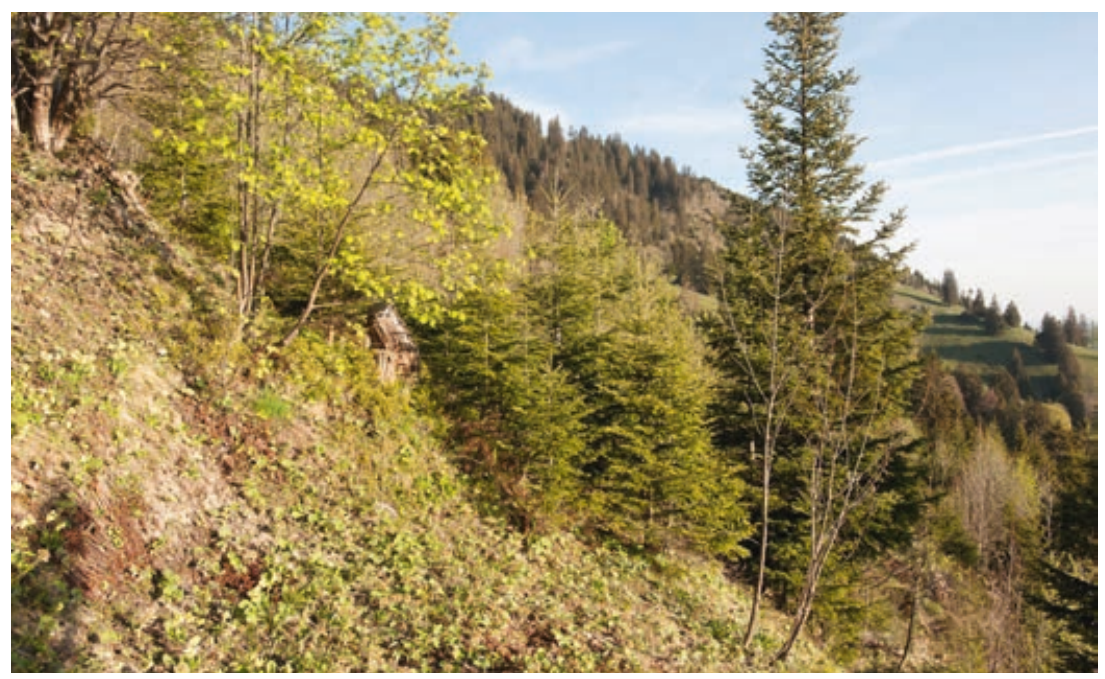

Abb 3 Die Fläche Pfäfers, Höfwald, am 7. Mai 2011. Dank (Tannen-)Vorverjüngung unter Schirm hat sich diese Vivianfläche rasch wiederbewaldet. Foto: Raphael Schwitter
Erwähnenswert ist die Behandlungsoption «Belassen mit Pflanzung». Sie ist in der Entscheidungshilfe bei Sturmschäden im Wald (BAFU 2008) nicht vorgesehen, da eine Bepflanzung im belassenen Sturmholz als nicht praxistauglich erachtet wurde. Beim Objekt «Tischli» in Pfäfers wurde sie trotzdem gewählt. Dank der Wirkung des liegenden Holzes konnte der Bau von Schneerechen vermieden werden (Schwitter 2011). Diese Option dürfte bei zukünftigen Sturmereignissen vermehrt Beachtung finden.

Auf weniger steilen Hängen kann auch die Option «Räumen und Bepflanzen» zum Erfolg führen. 24 Jahre später ist die Verjüngung auf bepflanzten Flächen deutlich weiter entwickelt als auf vergleichbaren unbepflanzten Flächen. Besonders deutlich zeigt sich dies im Chimichopfwald in Pfäfers. Auf einer bepflanzten Teilfläche ist die Schutzwirkung nahezu wiederhergestellt, während die natürliche Verjüngung auf einer unbepflanzten Teilfläche immer noch durch die Hochstauden bedrängt wird. Wichtig ist, dass die Bepflanzung in Kollektiven erfolgt, und dies bevor sich die Vegetationskonkurrenz entwickeln kann. Dadurch können erhebliche Einsparungen bei der Pflege erzielt werden.

Für die Praxis von grösster Bedeutung ist jedoch die ausreichende Präsenz von Verjüngung im Altbestand. Es ist wohl bezeichnend für den Zustand vieler Gebirgswälder, dass auf den meisten Viviansturmflächen zu wenig solcher Vorverjüngung für eine ausreichende Wiederbewaldung vorhanden war (Wohlgemuth \& Kramer 2015, dieses Heft). Nur auf einer von sechs Flächen, welche nach dem Sturm geräumt worden waren, entwickelte sich innerhalb von 24 Jahren eine schutzwirksame Bestockung aus Naturverjüngung (Tabelle 2): Auf der Fläche «Höfwald» in Pfäfers wuchs dank der Präsenz von Tannenansamung unter dem Schirm des Altbestandes ein Stangenholz in der gewünschten Baumartenzusammensetzung heran (Abbildung 3). In der Entscheidungshilfe bei Sturmschäden im Wald (BAFU 2008) gilt vorhandene Verjüngung, welche vom liegenden Holz «befreit» werden sollte, denn auch als Argument für die Räumung. Für die Massnahmenkombination «Belassen und Naturverjüngung» gibt es leider kein Beispiel mit genügend Vorverjüngung im Ausgangsbestand, das uns Aufschluss geben könnte über die Entwicklung von vorhandener Naturverjüngung im liegenden Holz.

\section{Die Entwicklung behindernde Faktoren}

In sieben der 16 beobachteten Objekte ist die Schutzwirkung 24 Jahre nach dem Sturm noch deutlich ungenügend (Tabelle 2). Im bereits erwähnten Beispiel «Bläserberg» in Pfäfers (geräumt, verbaut und bepflanzt) liegt es vor allem an den schwierigen Standortverhältnissen, die zu einem sehr langsamen Aufwachsen der gepflanzten Bäume führt. Auf vier 
von den fünf Flächen, die geräumt und anschliessend der natürlichen Wiederbewaldung überlassen wurden, genügt die Bestockungsdichte 24 Jahre nach dem Sturm nicht. Aus der spärlichen Vorverjüngung konnte kein Jungwald mit ausreichendem Deckungsgrad heranwachsen, und zusätzliche Naturverjüngung hat sich noch zu wenig eingestellt. Auch die durch die Räumungsarbeiten bewirkte vorübergehende Verbesserung des Keimbeetes hat dafür nicht ausgereicht. Die Gründe für das Ausbleiben der Naturverjüngung auf grossen Sturmflächen sind vor allem das Fehlen von Samenbäumen sowie die Konkurrenzvegetation, welche sich stark entwickelt und auch 24 Jahre nach dem Ereignis noch verjüngungshemmend wirkt. Leider fehlt infolge der Räumung dieser Flächen nun auch das Moderholz für den weiteren Verjüngungsprozess.

Etwas besser sieht die Zukunft auf den zwei Flächen «Pfäfers, Matongatter» und «Disentis, Cavorgia Mitte» aus, wo das Sturmholz liegen blieb. Hier steht der Fichten-Naturverjüngung in Zukunft ein günstiges Substrat zur Verfügung. Dazu muss das Holz allerdings mindestens 20 Jahre lang vermodern, wie auch andere Studien in Vivianflächen (Netzer 2010) und im Urwald Scatlè (Stroheker et al 2014) sowie die repräsentative Sturmflächen-Stichprobe (Wohlgemuth \& Kramer 2015, dieses Heft) zeigen. Mit der Zersetzung des Holzes nimmt allerdings auch dessen Wirkung gegenüber Schneebewegungen ab (Bebi et al 2015, dieses Heft).

Beim Objekt «Tschaler West» bei Curaglia hat sich die Bepflanzung nach der Räumung zwar gut entwickelt. Es entstand jedoch eine lange Phase mit ungenügender Schutzwirkung, was nur dank dem geringen Schadenpotenzial in Kauf genommen werden konnte. Eine ähnliche Situation liegt beim Objekt «Cavorgia Süd» bei Disentis vor. Zudem ist auf diesem blockigen Standort das Wachstum der jungen Bäume langsamer. Die Bepflanzung der verbauten Fläche im Objekt «Uaul Bugnei oben» im Tujetsch hat sich ähnlich gut entwickelt wie in den verbauten Flächen «Uaul Bugnei unten» und «Curaglia Stavel». Die Dichte der Bestockung genügt allerdings für einen ausreichenden Schutz noch nicht. Beim Objekt «Grot» in Pfäfers wurden Streuschäden liegen gelassen. Im Mischbestand aus Tanne und Fichte ist kein nennenswerter Käferbefall aufgetreten. Das liegende Holz hat auf dem grasreichen Standort einen Beitrag zur Reduktion des Schneegleitens geleistet. Dadurch konnte der Bau von Dreibeinböcken reduziert respektive zeitlich hinausgeschoben werden.

\section{Handlungsbedarf}

Die 16 Objekte wurden auch bezüglich Handlungsbedarf aus heutiger Sicht beurteilt. Insbesondere bei ungenügender Bestockung stellt sich die
Frage, ob Nachpflanzungen wirksam und verhältnismässig sind. Dabei wird befürchtet, dass die Vegetationskonkurrenz einen erheblichen Pflegeaufwand nach sich ziehen wird, der nur in wichtigen Schutzwäldern zu rechtfertigen ist. Dort müssen punktuelle Nachpflanzungen an den exponiertesten Stellen, allenfalls verbunden mit dem Ersatz einzelner Bauwerke, in Betracht gezogen werden. Damit wird indirekt auch bestätigt, dass es zweckmässig ist, wichtige Flächen frühzeitig und in ausreichendem Masse zu bepflanzen.

In mehreren der beurteilten Flächen wurde deutlich, dass es vorteilhafter gewesen wäre, mehr Holz liegen zu lassen. Hingegen wurde das liegende Holz an keinem Beispiel als problematisch beurteilt. Eine Gruppe kam an einem Objekt gar zum Schluss, dass der Räumungsentscheid aus heutiger Sicht nicht nachvollziehbar sei. Diese Aussage ist bezeichnend für die zunehmende Bereitschaft, das Holz gegebenenfalls an Ort und Stelle zu belassen.

\section{Folgerungen}

\section{Empfehlungen für die Waldbehandlung vor dem Sturm}

Mehr Verjüngung im Altbestand beziehungsweise mehr Vorverjüngung auf der Sturmfläche würde die Wiederbewaldung von Sturmflächen in Gebirgswäldern stark beschleunigen. In ihrem Verbreitungsgebiet leistet die schattenertragende Weisstanne einen wesentlichen Beitrag dazu. Werden reich strukturierte Bestände mit gut verteilter Verjüngung von Stürmen betroffen, sind die Aussichten für einen günstigen Verlauf der Wiederbewaldung eindeutig besser. Die Förderung von genügend und gut verteilten Verjüngungsansätzen sollte deshalb noch stärker beachtet werden, nicht nur bei der Pflege der Schutzwälder, sondern auch generell bei der Nutzung der Gebirgswälder.

\section{Empfehlungen für das Vorgehen nach dem Sturm}

Nach ausgedehnten Sturmschäden lohnt es sich, möglichst schnell ein Konzept für das Vorgehen zu erarbeiten, das auch die längerfristige Entwicklung der Schadenflächen berücksichtigt. Im Gebirgswald ausschlaggebend ist dabei das Vorgehen in Bezug auf Borkenkäfer. Die wissenschaftlichen Untersuchungen seit Vivian haben gezeigt, dass der Erfolg der Borkenkäferbekämpfung nach Sturmschäden nur in Streuschadengebieten relativ sicher ist (Forster \& Meier 2008). Es ist deshalb wichtig, sich nach Sturmschäden möglichst rasch einen Überblick über die Schadensituation zu verschaffen und das Vorgehen für einzelne Geländekammern differenziert nach Waldfunktionen festzulegen (siehe hierzu auch Meier-Glaser et al 2015, dieses Heft). Das ver- 


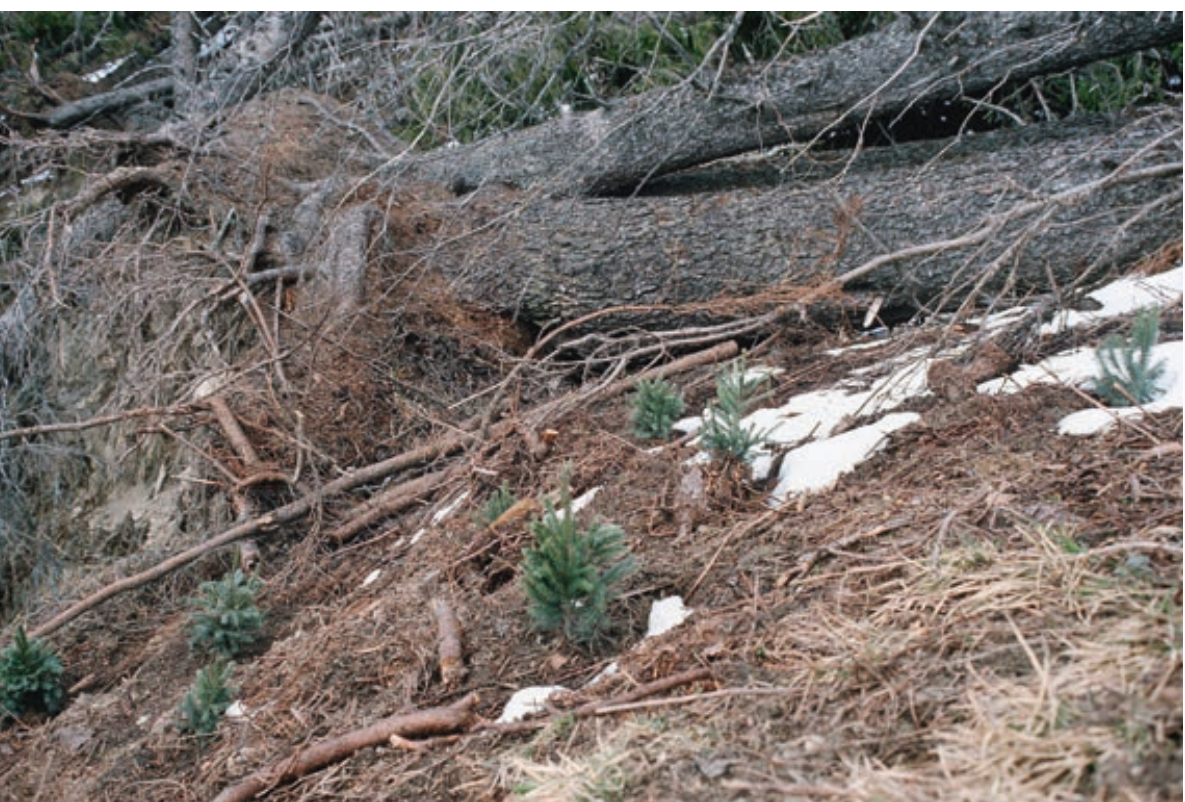

Abb 4 Pflanzung zwischen den liegenden Stämmen einer ungeräumten Fläche (Aufnahme vom 27. April 1991). Foto: Raphael Schwitter

schafft dem Forstdienst auch die Zeit, Totalschadenflächen individuell zu beurteilen und die langfristig erfolgreichen Handlungsoptionen zu bestimmen.

Die Wirtschaftlichkeit ist ein wichtiges Argument für oder gegen Räumung. Dabei müssen aber neben der Kostendeckung der Holzernte auch die Auswirkungen auf die Wiederbewaldung und die Kosten für bauliche Massnahmen berücksichtigt werden. Die Option «Belassen» ist vor allem dann sinnvoll, wenn so technische Massnahmen (Rundholzschneerechen, Gleitschneeverbau) eingespart werden können und wenn Moderholz für den Verjüngungsprozess notwendig ist.

Fehlt auf grossen Sturmflächen der hochmontanen und subalpinen Stufe die Vorverjüngung, dauert die Wiederbewaldung durch Naturverjüngung deutlich länger als die Phase, während der liegendes Holz genügend Schutz bieten kann. Mit der Option «Belassen mit Pflanzung» (Abbildung 4) kann in solchen Situationen die Phase mit einem Schutzdefizit deutlich verkürzt oder ganz überbrückt werden. $\mathrm{Zu}$ beachten ist bei dieser Option auch die Bedeutung des Schadenpotenzials. So liegt unterhalb des erwähnten Beispiels «Tischli» eine Verbindungsstrasse, die bei Gefahr gesperrt werden kann.

\section{Offene Fragen}

Die oben geschilderten Erkenntnisse und Folgerungen beruhen weitgehend auf Beobachtungen der Entwicklung von Einzelflächen unter immer wieder anderen Rahmenbedingungen. Zur Vertiefung der Erkenntnisse wäre es deshalb empfehlenswert, diese vergleichenden Beobachtungen auch auf weitere Sturmflächen und insbesondere solche auf anderen Waldstandorten auszudehnen. Gemäss den
Diskussionen an der Wintertagung der GWG im Februar 2014 interessieren dabei besonders die in Tabelle 3 genannten Fragen.

\section{Ausblick}

Die gestellten Fragen könnten mit einer Kombination von zusätzlichen Auswertungen vorhandener Daten, Zusatzstudien und Expertenworkshops bearbeitet werden. Mindestens Teilantworten sind von einem solchen Vorgehen zu erwarten.

Aufgrund der grossen Bedeutung der Vorverjüngung auf Sturmflächen stellt sich auch die Frage, inwieweit die Rahmenbedingungen für die Verjüngung insbesondere der Schutzwälder verbessert werden können. Mit den verfügbaren Mitteln sollten die minimalen Anforderungen auf möglichst grosser Fläche erfüllt werden.

Zwei Hauptresultate der GWG-Sommertagung 2014 sind hervorzuheben. Das erste ist eine gewisse Ernüchterung, was das Potenzial der Naturverjüngung auf grossen Sturmflächen betrifft. Das Räumen der Flächen ohne Pflanzung dürfte oft zu vorübergehend ungenügender Schutzwirkung führen, was nur in Wäldern ohne grosses Schadenpotenzial vertretbar ist. Das zweite Hauptresultat war, dass auf Sturmflächen noch differenzierter vorzugehen ist als bisher und als in der Entscheidungshilfe für Sturmschäden im Wald (BAFU 2008) dargestellt. Die Handlungsoptionen haben sich erweitert; es scheint heute auch zweckmässig, Pflanzungen und Räumungen in unterschiedlichen Anteilen zu kombinieren. Zum Beispiel sind Teilräumungen denkbar, und in belassenen Sturmflächen kann man stellenweise pflanzen. Die Voraussetzungen für die Anwendung dieser Handlungsoptionen sind nun genauer zu beschreiben und den forstlichen Entscheidungsträgern bekannt zu machen - denn der nächste Sturm kommt bestimmt.

Eingereicht: 22. September 2014, akzeptiert (mit Review): 22. Februar 2015

\section{Literatur}

BAFU (2008) Entscheidungshilfe bei Sturmschäden im Wald. Vollzugshilfe für die Wahl der Schadensbehandlung im Einzelbestand. Bern: Bundesamt Umwelt, Umwelt-Vollzug 0801. 132 p. BEBI P, PUTALLAZ JM, FANKHAUSER M, SCHMID U, SCHWITTER R ET AL (2015) Die Schutzfunktion in Windwurfflächen. Schweiz Z Forstwes 166: 168-176. doi: 10.3188/szf.2015.0168

BENZ G, ZUBER M (1997) Die wichtigsten Forstinsekten der Schweiz und des angrenzenden Auslandes. Zürich: VDF. 121 p. BRANG P, HILFIKER S, WASEM U, SCHWYZER A, WOHLGEMUTH T (2015) Langzeitforschung auf Sturmflächen zeigt Potenzial und Grenzen der Naturverjüngung. Schweiz Z Forstwes 166: 147-158. doi: 10.3188/szf.2015.0147

BROGGI MF (1990) Wald, Sturm, Aufrüsten und Borkenkäfer-Gedanken hierzu einige Zeit danach. Schweiz Z Forstwes 141: 1037-1044. 


\section{Windwurfanfälligkeit von Beständen}

Welche Möglichkeiten bestehen zur Reduktion der Windwurfanfälligkeit? Welche waldbaulichen Eingriffe erhöhen die Windwurfgefährdung?

Hanewinkel et al (2015, dieses Heft)

\section{Wiederkehrdauer von Windwürfen}

Wie sind die Prognosen für die Wiederkehrdauer von verheerenden Winterorkanen?

Usbeck (2015, dieses Heft)

\section{Nach dem Sturm}

\section{Streuschadenflächen}

Natürliche Verjüngung

In welchen Fällen ist mit ungenügender natürlicher Verjüngung zu rechnen?

Borkenkäfer

Wie kann Borkenkäferbefall in Flächen mit Streuschäden vermieden werden?

Meier-Glaser et al (2015

dieses Heft)

\section{Totalschadenflächen}

\section{Tun und Lassen}

Welche Kombination von Massnahmen, d.h. Räumen bzw. Liegenlassen und Pflanzen, führt im Einzelfall zu einer optimalen, kostengünstigen Überbrückung der vorübergehend abnehmenden Schutzwirkung?

Welches Vorgehen wird bei Teilräumungen empfohlen (Arbeitssicherheit, Arbeitsablauf, Holzernteverfahren etc.)?

Zu welchen Anteilen wird die vorhandene Verjüngung durch Räumung reduziert bzw. beschädigt? In welchem

Ausmass wird etablierte Verjüngung durch sich bewegende liegen gelassene Stämme beschädigt?

\section{Natürliche Verjüngung}

Lässt sich das Verjüngungspotenzial auf Windwurfflächen einschätzen, bzw. kann mittels weniger Indikatoren (z.B. zur Stammzahl und Deckung der Verjüngung) über eine Ergänzungspflanzung entschieden werden?

Auf welchen Standorten ist eine natürliche Verjüngung durch Begleitvegetation oder andere Faktoren besonders limitiert, auf welchen kommt sie besonders gut an?

\section{Pflanzung, Jungwaldpflege}

Wie können Fichtensamen erfolgreich auf Moderholz eingebracht werden, und wann ist der geeignete Zeitpunkt dafür (beim Fehlen von Samenbäumen in grossen Sturmschadenflächen)?

Welche Baumarten sollen im Hinblick auf den Klimawandel bei Pflanzungen und bei der Jungwaldpflege bevorzugt werden?

\section{Massenbewegungen}

Wie wirken sich liegen gelassene Bäume auf die Bodenerosion und flachgründige Rutschungen aus?

Gibt es kostengünstige Alternativen zu teuren Rundholzschneerechen, um die vorübergehend kritische Schutzwirkung zu überbrücken?

Wie lassen sich die Naturgefahrenprozesse auf Sturmflächen besser vorhersagen? Können die anfängliche Schutzwirkung von liegendem Holz und deren Entwicklung einfach quantifiziert werden (z.B. Diagramm mit Wirkhöhen, Verteilung, Dichte, Zersetzungsgrad etc.)?

\section{Borkenkäfer}

Können Nachbarbestände von Sturmschadenflächen wirksam vor Käferbefall geschützt werden (Modell für Aufbau und Ausbreitung von Massenvermehrungen)?

Tab 3 Fragen zum Auftreten und zur Behandlung von Windwürfen, auf der Basis von Ideen aus der GWG-Wintertagung entwickelt.

FEISTL T, BEBI P, DREIER L, HANEWINKEL M, BARTELT P (2014) Quantification of basal friction for technical and silvicultural glide-snow avalanche mitigation measures. Nat Hazards Earth Syst Sci 14: 2921-2931.

FORSTER B, MEIER F (2008) Sturm, Witterung und Borkenkäfer: Risikomanagement im Forstschutz. Birmensdorf: Eidgenöss Forsch.anstalt WSL, Merkbl Prax 44. 8 p.

FREHNER M, WASSER B, SCHWITTER R (2005) Nachhaltigkeit und Erfolgskontrolle im Schutzwald. Wegleitung für Pflegemassnahmen in Wäldern mit Schutzfunktion. Bern: Bundesamt Umwelt Wald Landschaft. 564 p.

GWG (2014) Dokumentation zur Tagung «Waldsukzession und Naturgefahrenprozesse auf Sturmflächen». Maienfeld: Schweizerische Gebirgswaldpflegegruppe. www.gwg-gsm.ch/tl_ files/gebirgswald/de/04_GWG/GWG_Tagungen/2014/Dokumentation\%20GWG-Wintertagung\%202014.pdf (23.2.2015).
Frehner et al (2005)

z.B. Leuenberger (2003),

Bebi et al (2015, dieses Heft)

Feistl et al (2014)
HANEWINKEL M, ALBRECHT A, SCHMIDT M (2015) Können Windwurfschäden vermindert werden? Eine Analyse von Einflussgrössen. Schweiz Z Forstwes 166: 118-128. doi: 10.3188/ szf.2015.0118

LEUENBERGER F (2003) Bauanleitung Gleitschneeschutz- und temporärer Stützverbau. Davos: WSL-Institut für Schnee und Lawinenforschung. 97 p.

MEIER-GLASER AL, BEER W, MARTI W (2015) Erfahrungen mit Windwurf im Kanton Bern. Schweiz Z Forstwes 166: 177-183. doi: 10.3188/szf.2015.0177

MEYER-GRASS M, IMBECK H (1985) Waldlawinen: gefährdete Bestände, Massnahmen. Davos: Eidgenöss Institut SchneeLawinenforschung, Merkbl. 6 p.

NETZER V (2010) Langfristige Lawinenschutzwirksamkeit auf Windwurfflächen: Entwicklung der Verjüngung. Zürich: ETH Zürich, Masterarbeit. 49 p. 
OTT E (1972) Erhebungen über den gegenwärtigen Zustand des Schweizer Waldes als Grundlage waldbaulicher Zielsetzungen. Mitt Eidgenöss Anst Forstl Versuchswes 48: 1-193.

SCHÖNENBERGER W (2002) Windthrow research after the 1990 storm Vivian in Switzerland: objectives, study sites, and projects. For Snow Landsc Res 77 (1/2): 9-16.

SCHWITTER R (1996) Schutzwald im Taminatal - Wiederherstellung nach dem Sturm. Forstwiss Cent.bl 115: 273-286.

SCHWITTER R (2011) 20 Jahre nach dem Sturm. Sturmholz als Lawinenschutz - ein Erfahrungsbericht. Wald Holz 92 (6): 29-31.

STROHEKER S, MARTIN S, SIEBER T, BUGMANN H, WEISS M (2014) Welche Faktoren bestimmen den Erfolg der Moderholzverjüngung im Fichtenurwald Scatlè? Schweiz Z Forstwes 165: 339-347. doi: 10.3188/szf.2014.0339

USBECK T (2015) Wintersturmschäden im Schweizer Wald von 1865 bis 2014 . Schweiz Z Forstwes 166: 184-190. doi: 10.3188/ szf.2015.0184
WALCHER J (1991) Sturmschäden 1990: eine Lagebeurteilung aus der Sicht der Praxis: Beispiele aus dem Kanton Glarus. Schweiz Z Forstwes 142: 463-469.

WANGLER F (1974) Die Sturmgefährdung der Wälder in Südwestdeutschland. Eine waldbauliche Auswertung der Sturmkatastrophe 1967. Freiburg i. Br.: Albert-Ludwigs Univ Freiburg, Dissertation. $226 \mathrm{p}$.

WOHLGEMUTH T, KRAMER K (2015) Waldverjüngung und Totholz in Sturmflächen 10 Jahre nach Lothar und 20 Jahre nach Vivian. Schweiz Z Forstwes 166: 135-146. doi: 10.3188/ szf.2015.0135

WSL, BUWAL (2001) Lothar. Der Orkan 1999. Ereignisanalyse. Birmensdorf: Eidgenöss Forsch.anstalt WSL. 365 p.

ZELLER E (1977) Pflege von Fichtenaufforstungen im Gebirge. Bündnerwald 30: 197-202.

\section{Leçons tirées de Vivian pour les forêts de montagne - en vue du prochain ouragan}

L'ouragan «Vivian» de février 1990 a surpris l'économie forestière suisse par son énorme ampleur et a soulevé des questions qui n'avaient encore jamais été abordées. Cela présentait donc un grand défi tant pour les chercheurs que pour les praticiens. 24 ans après la tempête, le Groupe suisse de sylviculture de montagne a analysé, lors de deux réunions, l'évolution de la régénération et son effet protecteur contre les dangers naturels dans plusieurs surfaces bien documentées, ravagées par Vivian et situées entre 1500 et 1700 m dans les cantons de St-Gall et des Grisons. La décision de déblayer les parterres de coupe ou de laisser le bois sur place dépend, en premier lieu, du risque d'une attaque par les bostryches. Les arbres couchés peuvent diminuer temporairement les risques de dangers naturels et favoriser le reboisement car ils diminuent les glissements de neige et forment, à long terme, du bois décomposé. Après 24 années, seules quelques surfaces étudiées présentaient un niveau de reboisement assez conséquent pour assurer, à nouveau, un effet protecteur suffisant. Cependant, la majorité des surfaces n'ont toujours pas atteint ce niveau de reboisement. La présence de régénération préétablie dans un peuplement antérieur accélère considérablement le reboisement de la surface de chablis. En absence d'une telle régénération préétablie, une plantation peut servir à atteindre une avance importante sur la régénération naturelle qui s'installe seulement peu à peu. En résumé, il est à constater que sur de vastes surfaces de chablis situées à haute altitude, le potentiel de régénération naturelle est limité, et que, lors de chablis futurs, il faudrait combiner de manière plus variée les différentes options de gestion.

\section{Lessons from Vivian for mountain forests - regarding the next storm}

The winter storm "Vivian" in February 1990 took Swiss forestry by surprise due to its enormous extent. It gave cause for entirely new and challenging questions for scientists and practitioners. Twenty-four years after the storm, the Swiss mountain forest tending group assessed in the frame of two workshops forest succession and the evolution of the protective effect against natural hazards in several well-documented Vivian storm areas at elevations between 1,500 and 1,700 m a.s.I. in the cantons of St. Gallen and Grisons. The decision to salvage the damaged timber or to leave it in place depends primarily on how the risk of bark beetle infestations is assessed. Lying stems can transiently reduce the risk created by natural hazards and favor stand establishment, by reducing snow movements and by serving as nurse logs in the long term. On several areas among those studied, forest succession has progressed so far that the protective effect is nearly restored. However, in the majority of the areas, this is not yet the case even 24 years after the storm. The presence of regeneration in the pre-storm stand, so-called advance regeneration, considerably accelerates forest succession in a windthrow area. If advance regeneration is absent, planting can considerably fasten succession in comparison to natural regeneration, which establishes often slowly. In summary, it is concluded that on large windthrow areas at high elevation the potential of natural regeneration is limited and that a more varied combination of the different treatment options should be envisaged in the case of future windthrow events. 